\title{
ON THE DICHOTOMY PROBLEM FOR TENSOR ALGEBRAS
}

BY

\author{
J. BOURGAIN
}

\begin{abstract}
Let $I, J$ be discrete spaces and $E \subset I \times J$. Then either $E$ is a $V$-Sidon set (in the sense of $[2, \S 11]$ ), or the restriction algebra $A(E)$ is analytic. The proof is based on probabilistic methods, involving Slépian's lemma.
\end{abstract}

1. Introduction and definitions. A subset $E$ of $I \times J$ is called a $V$-Sidon set provided the restriction of $l^{\infty}(I) \hat{\otimes} l^{\infty}(J)$ coincides with $l^{\infty}(E)$. It is known then that $E$ is obtained as the finite union of "sections" $F \subset I \times J$, meaning that either $\left.\pi_{1}\right|_{F}$ or $\left.\pi_{2}\right|_{F}$ is one-to-one $\left(\pi_{1}, \pi_{2}\right.$ respective coordinate projections). Our purpose is to show that the algebra $A(E)$, obtained by restricting $l^{\infty}(I) \hat{\otimes} l^{\infty}(J)$ to $E$, is either $l^{\infty}(E)$ or analytic. Recall that an algebra is analytic provided that only analytic functions operate on it (see [2] for more details). In view of Malliavin's characterization of analytic algebras, it amounts to showing the following (see [2, p. 102]).

THEOREM. If $E \subset I \times J$ is not a $V$-Sidon set, then for some $c>0$

$$
\sup _{\substack{\|\phi\|_{A(E)} \leqslant 1 \\ \phi \text { real }}}\left\|e^{i t \phi}\right\|_{A(E)}>e^{c t}, \quad t>0 .
$$

In fact, $c$ will be an absolute constant.

2. A condition for analyticity. In this section, a criterion is explained which permits us to minorize $\left\|e^{i t \phi}\right\|_{A(E)}$. Let $f_{z}$ stand for the translate of $f$ by $z$.

LEMMA 1. Let $G$ be a compact Abelian group and $E$ be a subset of the dual group $\Gamma$ of $G$. Denote by $C_{E}$ the space of continuous functions with Fourier transform supported by $E$. Fix a positive integer and assume the existence of a function $f$ in $C_{E}$ and a sequence of points $x_{1}, \ldots, x_{1}$ in $G$ satisfying

$$
\begin{gathered}
f(0)=\|f\|_{\infty}=1, \\
\sum_{S \subset\{1, \ldots, l\}}\left|f_{\Sigma_{k} \in S} x_{k}\right| \leqslant B \text { pointwise on } G .
\end{gathered}
$$

$\left(\sum_{S} x_{k}\right.$ refers to the group operation in $G$.) Then $(c=$ numerical $)$

$$
\sup _{\substack{\|\phi\|_{A(E)} \leqslant 1 \\ \phi \text { real }}}\left\|e^{i t \phi}\right\|_{A(E)} \geqslant e^{c t} \quad \text { if } B<t<l .
$$

Received by the editors March 30, 1985.

1980 Mathematics Subject Classification. Primary 42A55, 43A46, 43A75, 43A10.

(C)1986 American Mathematical Society $0002-9947 / 86 \$ 1.00+\$ .25$ per page 
Proof. Define $\sigma(t)$ to be the left member of (3). From the simple estimation, valid in any Banach algebra $A$,

$$
\left\|\prod_{k}\left(1+u_{k}\right)\right\|_{A} \leqslant e^{\Sigma\left\|u_{k}\right\|^{2}} \sup _{S}\left\|e^{\sum_{s} u_{k}}\right\|, \quad\left\|u_{k}\right\|<1 / 2
$$

applied to the elements

$$
u_{k}=\frac{i t}{4 l} \varepsilon_{k}\left[\left.\left(1-i \varepsilon_{k}^{\prime}\right) \hat{\delta}_{x_{k}}\right|_{E}+\left.\left(1+i \varepsilon_{k}^{\prime}\right) \hat{\delta}_{-x_{k}}\right|_{E}\right] \quad\left(i^{2}=-1\right)
$$

it follows that

$$
\begin{gathered}
\left\|\prod_{1 \leqslant k \leqslant l}\left[1+\frac{i t}{4 l} \varepsilon_{k}\left[\left.\left(1-i \varepsilon_{k}^{\prime}\right) \hat{\delta}_{x_{k}}\right|_{E}+\left.\left(1+i \varepsilon_{k}^{\prime}\right) \hat{\delta}_{-x_{k}}\right|_{E}\right]\right]\right\|_{A(E)} \\
\leqslant 2 e^{t^{2} / l} \sigma(t) .
\end{gathered}
$$

Here $\varepsilon \in\{1,-1\}^{\prime}, \varepsilon^{\prime} \in\{1,-1\}^{\prime}$ will be used in an averaging argument. Let $\left\{d_{S} \mid S\right.$ $\subset\{1, \ldots, l\}\}$ be elements of the unit disc. From the $C_{E}-A(E)$ norm duality and (2), the following minoration for the left member of (4) is valid ( $w_{S}$ refers to the usual Walsh system):

$$
\begin{aligned}
\frac{1}{B} \int & \left|\left\langle\prod_{1 \leqslant k \leqslant l}\left[1+\frac{i t}{4 l} \varepsilon_{k}(\cdots)\right], \sum_{S} d_{S} w_{S}(\varepsilon) f_{\Sigma_{S} x_{k}}\right\rangle\right| d \varepsilon \\
& \geqslant \frac{1}{B} \mid \sum_{S \subset\{1, \ldots, l\}} d_{S}\left(\frac{i t}{4 l}\right)^{|S|}\left\langle\underset{k \in S}{*}\left[\left(1-i \varepsilon_{k}^{\prime}\right) \delta_{x_{k}}+\left(1+i \varepsilon_{k}^{\prime}\right) \delta_{-x_{k}}\right], f_{\Sigma_{S} x_{k}}\right\rangle .
\end{aligned}
$$

For an appropriate choice of the $d_{S}=d_{S}\left(\varepsilon^{\prime}\right)$, the identity

$$
\int\left\{\underset{k \in S}{*}\left[\left(1-i \varepsilon_{k}^{\prime}\right) \delta_{x_{k}}+\left(1+i \varepsilon_{k}^{\prime}\right) \delta_{-x_{k}}\right]\right\}\left(\prod_{k \in S} \frac{1+i \varepsilon_{k}^{\prime}}{2}\right) d \varepsilon^{\prime}=\delta_{\Sigma_{s} x_{k}}
$$

and integration in $\varepsilon^{\prime}$ lead to the minoration

$$
\sum_{S \subset\{1, \ldots l l\}} B^{-1}\left(\frac{t}{2 \sqrt{2} l}\right)^{|S|}\left|\left\langle f_{\Sigma_{s} x_{k}}, \delta_{\sum_{S} x_{k}}\right\rangle\right|=\left(1+\frac{t}{2 \sqrt{2} l}\right)^{\prime} \frac{1}{B}
$$

as a consequence of $(1)$. Hence $\sigma(t) \geqslant(1 / B) e^{-t^{2} / l} \cdot e^{c_{1} t}$, and the result easily follows.

REMARK. To satisfy (1), (2) is possible only if $C_{E}$ contains $l_{k}^{\infty}$-subspaces of arbitrary large dimension $k$ (in the Banach space sense). Hence, a natural question is the "cotype-dichotomy" problem (explained in [4]). This conjecture was recently solved in the affirmative (see [1]), and implies that if $E$ is not a Sidon set, then

$$
\sup _{\substack{\|\phi\|_{A(E)} \leqslant 1 \\ \phi \text { real }}}\left\|e^{i t \phi}\right\|>c t, \quad \forall t>0 .
$$

3. Verification of the condition in the tensor algebra case. It remains to prove that if $E \subset I \times J$ is not a $V$-Sidon set, then (1), (2) of Lemma 1 can be realized. In this case, let $G$ be a Cantor-group $\{1,-1\}^{\mathbf{N}} \times\{1,-1\}^{\mathbf{N}}$ and identify $I$ (resp. $J$ ) with the 
Rademacher sequence $\alpha_{i}(x)$ (resp. $\left.\beta_{j}(y)\right)$ on the first (resp. second) factor $(i, j=$ $1, l, \ldots)$. The following well-known (and easy) combinatorial lemma is applied to $E$ (see $[2,11.8 .1])$.

LEMMA 2. If $E \subset I \times J$ is not a $V$-Sidon set, then for arbitrary $K$ there are finite subsets $I_{1} \subset I$ and $J_{1} \subset J$ (say $\left.\left|I_{1}\right| \geqslant\left|J_{1}\right|\right)$, and for each $i \in I_{1}$ a subset $A_{i} \subset J_{1}$, $\left|A_{i}\right|=K$, satisfying $\bigcup_{i \in I_{1}}\left(\{i\} \times A_{i}\right) \subset E$.

With those notations, let

$$
f=\sum_{i \in I_{1}} \sum_{j \in A_{1}} \alpha_{i} \otimes \beta_{j}
$$

Thus $f(0)=K\left|I_{1}\right|=\|f\|_{\infty}$. The realization of (1), (2) above will be clear from

Lemma 3. Let $2^{l}<K^{1 / 4}$. Then, as for an absolute constant $C$,

$$
\int_{G}\left\|\sum_{S \subset\{1, \ldots, l\}}\left|f_{\Sigma_{S z_{k}}}\right|\right\|_{\infty} d z_{1} \cdots d z_{l} \leqslant C K\left|I_{1}\right|
$$

( $G^{\prime}$ is the l-fold product $G \times \cdots \times G$ with normalized measure.)

Proof. Write $z \in G \equiv\{1,-1\}^{\mathbf{N}} \times\{1,-1\}^{\mathbf{N}}$ as $z=(u, v)$. Thus

$$
f_{z}=\sum_{i} \sum_{j \in A_{i}} \alpha_{i}(u) \beta_{j}(v) \alpha_{i} \otimes \beta_{j} .
$$

For fixed $(x, y) \in G$, there are 1-bounded scalars $\left\{c_{S} \mid S \subset\{1, \ldots, l\}\right\}$ satisfying

$$
\begin{gathered}
\sum_{S}\left|f_{\sum_{S z_{k}}}(x, y)\right|=\left|\sum_{i \in I_{1}} \alpha_{i}(x) \sum_{S \subset\{1, \ldots, l\}} c_{S} \alpha_{i}\left(\sum_{S} u_{k}\right) \sum_{j \in A_{i}} \beta_{j}\left(\sum_{S} v_{k}\right) \beta_{j}(y)\right| \\
\leqslant\left|I_{1}\right|^{1 / 2}\left\{\sum_{i \in I_{1}}\left|\sum_{S} c_{S} \alpha_{i}\left(\sum_{S} u_{k}\right)\left\{\sum_{j \in A_{i}} \beta_{j}\left(\sum_{S} v_{k}\right) \beta_{j}(y)\right\}\right|^{2}\right\}^{1 / 2} .
\end{gathered}
$$

The second factor may be estimated by expanding the inner square as

$$
\begin{aligned}
& \left\{\sum_{i \in I_{1}} \sum_{S}\left|\sum_{j \in A_{i}} \beta_{j}\left(\sum_{S} v_{k}\right) \cdot \beta_{j}(y)\right|^{2}\right\}^{1 / 2} \\
& \quad+\left\{\sum_{S \neq S^{\prime}}\left|\sum_{i \in I_{1}} \alpha_{i}\left(\sum_{S \triangle S^{\prime}} u_{k}\right)\left\{\sum_{j \in A_{i}} \beta_{j}\left(\sum_{S} v_{k}\right) \beta_{j}(y)\right\}\left\{\sum_{j \in A_{i}} \beta_{j}\left(\sum_{S^{\prime}} v_{k}\right) \beta_{j}(y)\right\}\right|\right\}^{1 / 2}
\end{aligned}
$$

It remains to take the supremum over $y$. The first and second terms will be treated separately. 
First term in (6). Fix $i \in I_{1}$. Linearize the square function by considering scalars $\left\{a_{S} \mid S \subset\{1, \ldots, l\}\right\}, \Sigma\left|a_{S}\right|^{2}=1$ so that

$$
\begin{aligned}
\left(\left.\sum_{S}\left|\sum_{j \in A_{i}} \beta_{j}\left(\sum_{k \in S} v_{k}\right) \beta_{j}(y)\right|^{2}\right|^{1 / 2}\right. & =\left|\sum_{j \in A_{i}} \beta_{j}(y) \sum_{S} a_{S} \beta_{j}\left(\sum_{k \in S} v_{k}\right)\right| \\
& \leqslant\left|A_{i}\right|^{1 / 2}\left\{\sum_{j \in A_{i}}\left|\sum_{S} a_{S} \beta_{j}\left(\sum_{k \in S} v_{k}\right)\right|^{2}\right\}^{1 / 2}
\end{aligned}
$$

by the Cauchy-Schwarz inequality.

For fixed $j \in A_{i}$, expand the square. Reversing the order of summation yields the estimation

$$
\begin{aligned}
& \left|A_{i}\right|^{1 / 2}\left\{\left|A_{i}\right|+\sum_{S \neq S^{\prime}}\left|a_{S}\right|\left|a_{S^{\prime}}\right|\left|\sum_{j \in A_{i}} \beta_{j}\left(\sum_{k \in S \triangle S^{\prime}} v_{k}\right)\right|\right\}^{1 / 2} \\
& \leqslant\left|A_{i}\right|^{1 / 2}\left\{\left|A_{i}\right|+\left(\left.\sum_{S \neq S^{\prime}}\left|\sum_{j \in A_{i}} \beta_{j}\left(\sum_{k \in S \triangle S^{\prime}} v_{k}\right)\right|^{2}\right|^{1 / 2}\right\}^{1 / 2} .\right.
\end{aligned}
$$

This estimation is uniform on $G$ and depends only on $z_{1}, \ldots, z_{l}$. Since for $S \neq S^{\prime}$, $\left(v_{1}, \ldots, v_{l}\right) \mapsto \Sigma_{k \in S \Delta S^{\prime}} v_{k}$ gives the Haar measure on $\{1,-1\}^{\mathbf{N}}$ as the image measure, the integration w.r.t. $z_{1}, \ldots, z$, appearing in (5) yields the estimation for (7)

$$
\left|A_{i}\right|^{1 / 2}\left(\left|A_{i}\right|+2^{\prime}\left\|\sum_{j \in A_{i}} \beta_{j}\right\|_{2}\right)^{1 / 2} \leqslant K^{1 / 2}\left(K+2^{l} K^{1 / 2}\right)^{1 / 2}<2 K,
$$

by hypothesis on $l$ and $\left|A_{i}\right|=K$.

Second term in (6). Let $\left\{g_{i}(\omega) \mid i \in I_{1}\right\}$ denote a sequence of independent Gaussian variables on some probability space $\Omega$, and let $X_{i}(y)=\sum_{j \in A_{i}} \beta_{j}(y)$ be defined on $\{1,-1\}^{\mathbf{N}}$. The expressions

$$
\int \sup _{y}\left|\sum_{i \in I_{1}} \alpha_{i}\left(\sum_{S \Delta S^{\prime}} u_{k}\right) X_{i}\left(y \sum_{S} v_{k}\right) X_{i}\left(y \sum_{S^{\prime}} v_{k}\right)\right| d u_{1} \cdots d u_{l}
$$

are dominated by

$$
\int \sup _{y, y^{\prime} \in\{1,-1\}^{N}}\left|\sum_{i \in I_{1}} g_{i}(\omega) X_{i}(y) X_{i}\left(y^{\prime}\right)\right| d \omega .
$$

It follows from the inequality

$$
\begin{aligned}
& \left(\sum_{i \in I_{1}}\left|X_{i}(y) X_{i}\left(y^{\prime}\right)-X_{i}\left(y_{1}\right) X_{i}\left(y_{1}^{\prime}\right)\right|^{2}\right)^{1 / 2} \\
& \quad \leqslant K\left\{\sum_{I_{1}}\left|X_{i}(y)-X_{i}\left(y_{1}\right)\right|^{2}+\sum_{I_{1}}\left|X_{i}\left(y^{\prime}\right)-X_{i}\left(y_{1}^{\prime}\right)\right|^{2}\right\}^{1 / 2}
\end{aligned}
$$


and Slépian's comparison lemma for Gaussian processes [3] that (8) may be estimated by

$$
C K \int \sup _{y \in\{1,-1\}^{N}}\left|\sum_{I_{1}} g_{i}(\omega) X_{i}(y)\right| d \omega
$$

Since

$$
\left|\sum_{I_{1}} g_{i}(\omega) X_{i}(y)\right| \leqslant \sum_{j \in J_{1}}\left|\sum_{i \mid j \in A_{i}} g_{i}(\omega)\right|
$$

(9) is less than

$$
C K\left\{\sum_{j \in J_{1}}\left|\left\{i \mid j \in A_{i}\right\}\right|^{1 / 2}\right\} \leqslant C K\left|J_{1}\right|^{1 / 2}\left(\sum_{i \in I_{1}}\left|A_{i}\right|\right)^{1 / 2} \leqslant C K^{3 / 2}\left|I_{1}\right| .
$$

Therefore, (6) contributes to $C 2^{l} K^{3 / 4}\left|I_{1}\right|^{1 / 2}$. Collecting estimations, one concludes

$$
\int_{G^{\prime}}\left\|\sum_{S}\left|f_{\sum_{k \in S^{2} k}}\right|\right\|_{\infty} d z_{1} \cdots d z_{l} \leqslant C\left|i_{1}\right|^{1 / 2}\left(K\left|I_{1}\right|^{1 / 2}+2^{\prime} K^{3 / 4}\left|I_{1}\right|^{1 / 2}\right)<C K\left|i_{1}\right|
$$

since $l$ was chosen small enough. Hence (5) is proved.

4. Further remarks. (1) The result stated in the abstract can be generalized as follows: Let $k$ be a positive integer, $I_{1}, \ldots, I_{k}$ discrete spaces and $E \subset$ $\left(I_{1} \times \cdots \times I_{k}\right)$. Then either $E$ is a $V$-Sidon set, or the restriction algebra

$$
\left[l^{\infty}\left(I_{1}\right) \hat{\otimes} \cdots \hat{\otimes} l^{\infty}\left(I_{k}\right)\right] / E^{\perp}
$$

is analytic. The argument presented above can indeed be adapted to the case of several factors. This adaptation, however, requires some additional work. (Notice that the role of the factors $I$ and $J$ in the previous computation is different.)

(2) Let $F$ be a finite subset of the dual $\Gamma$ of a compact abelian group $G$. According to [5], call the arithmetical diameter $d(F)$ of $F$ the smallest number $d$ for which there exists a subset $P$ of the unit ball of $P M(F),|P|=d$, such that

$$
\|f\|_{\infty} \leqslant 2 \sup _{\mu \in P}|\langle f, \mu\rangle| \quad \text { if } f \in C_{F} .
$$

The method presented in this note permits us to show that for $E \subset \Gamma$ the restriction algebra $A(E)$ is analytic as soon as

$$
\varlimsup_{k} \sup _{\substack{F \subset E \\|F|=k}} \frac{(\log |F|)^{2}}{\log d(F)}=\infty
$$

improving on the sufficient condition obtained in [5]. Details will appear elsewhere.

(3) The verification "at random" of the dichotomy conjecture for Sidon sets [6] is possible by using the criterion presented in $\$ 2$ of this note. 


\section{REFERENCES}

1. J. Bourgain and V. D. Milman, La dychotomie du cotype pour les espaces invariants, C. R. Acad. Sci. Paris Sér. I Math. 300 (1985), 263-266.

2. G. Graham and C. McGehee, Essays in commutative harmonic analysis, Springer, New York, 1982.

3. X. Fernique, Regularite des trajectoires des fonctions aleatoires gaussiennes, Lecture Notes in Math., vol. 480, Springer, New York, 1975.

4. S. Kwapien and A. Pelczynski, Absolutely summing operators and translation invariant spaces of functions on compact abelian groups, Math. Nachr. 94 (1980), 303-340.

5. Y. Katznelson and P. Malliavin, Un critère d'analyticitè pour les algèbres de restriction, C. R. Acad. Sci. Paris Sér. A-B 261 (1965), A4964-A4967.

6. Verification statistique de la conjecture de la dichotomie sur une classe d' algèbres de restriction, C. R. Acad. Sci. Paris Sér. A-B 262 (1966), A490-A492.

Department of Mathematics, California Institute of Technology, Pasadena, California

Department of Mathematics, Vrije Universiteit, Pleinlaan 2.F7, 1050 - Brussels, Belgium 